Result Metrics on these efforts are publicly available thru Wikipedia, the Wiki Education Foundation and Altmetric so reach can be evaluated continuously by the number of views and quality of entries.

Discussion Several mechanisms exists to motivate researchers to get involved and to evaluate the impact of the efforts in Wikipedia. Throughout these initiatives, new scientific content related to acoustics was successfully added to Wikipedia, and the quality of the entries were improved.

\section{$1682 \mathrm{~g}$ VISION ZERO. WHAT IS IT? WHY SHOULD WE CARE? WHAT SHOULD WE DO?}

\section{S Timm. German Social Accident Insurance DGUV, Sankt Augustin, Germany}

\subsection{6/oemed-2018-ICOHabstracts.206}

Introduction 'Vision Zero' is a prevention strategy with the vision of a world without occupational accidents and workrelated diseases. Its highest priority is to prevent fatal and serious work accidents and occupational diseases. Vision Zero is the goal of a comprehensive culture of prevention.

Methods The concept of Vision Zero has gone hand in hand with the work done by the German Social Accident Insurance DGUV and its member institutions for many years. Vision zero in context with safety and health at work is not an illusion or a target of its own; it is a basic strategy and a mindset at the same time. Vision Zero is the fundamental strategy for developing a comprehensive and holistic culture of prevention that takes into consideration the safety, health and wellbeing of employees. The International Social Security Association ISSA just recently launched a global 'Vision Zero' campaign during the World Congress on Safety and Health at Singapore.

Results In order for Vision Zero to become a reality in practice, prevention work must always be realigned to this goal. This broad global and networked approach requires everyone in society to get involved. Vision Zero is a strategic, comprehensive and qualitative approach: where objectives are formulated and agreed upon; which is geared towards risks and hazards; and which takes into consideration all the circumstances behind accidents at work and on the road, occupational diseases and work-related health hazards.

Conclusions The goal of zero accidents might seem difficult, but it is the only ethically correct goal that we must work on in the future. Vision Zero provides a strategy to achieve this. Under Vision Zero, safety and health at work are values that companies, organisations and society appreciate and strive for.

\section{2h USING NEW METRICS TO EVALUATE DIGITAL COMMUNICATIONS IMPACT ON OSH STAKEHOLDER'S COMMUNITIES ONLINE}

\section{S Rouhi. Altmetric.com, London, UK}

\subsection{6/oemed-2018-ICOHabstracts.207}

Introduction Alternative metrics are any digital indicator of online behaviour around a web-native item - these can range from blog posts to comments to shares to download. Since launching in 2012, Altmetric.com collects online metrics and metadata around millions of digital research and communications outputs ranging from press releases to peer reviewed journal publications. Using automated and manual data gathering techniques, we have conducted sentiment and stakeholder affiliation analyses of data from the National Institutes for Occupation Health and Safety to help identify key stakeholder networks in the OSH community, how they engage, what platforms they use, and what they're saying.

Methods We reviewed NIOSH attention data across the 16 platforms Altmetric tracks using the Altmetric Explorer platform. We grouped those platforms into 'red flag' and 'green flag' platforms with distinct sentiment patterns. Red flag platforms were platforms were the sentiment was most likely to be negative or critical. Green flag platforms were platforms where qualitative engagement was most likely to be positive. Blue flag platforms had no sentiment and simply provided findings without editorialising about them.

Results NIOSH's break out using this flagging system was unique and broke out as follows. Red flags included blogs, Reddit, and peer review sites. News, Twitter, and Wikipedia tend to have non-editorialised reporting of NIOSH communications and solely need to be monitored for accuracy - blue flag platforms. Green flag platforms with high positive sentiment likelihood include F1000, Facebook, YouTube, policy and syllabi citations. Stakeholder analysis is ongoing of who engages on these platforms is ongoing and may not be ready for this presentation.

Conclusions Peer-reviewed OSH journal literature and webnative digital communications are underserved by existing metrics like citations and web analytics. New web-based alternative metrics and provide deep qualitative data on what communities are being reach, when, on what platforms, and what the content of their engagement is. These new metrics can play a vital role in shaping communications across $\mathrm{OSH}$ organisations globally

\section{2i FRAMEWORK FOR A SOCIAL MEDIA STRATEGY TO CREATE COMMUNITIES OF INTEREST AND REACH NON- TRADITIONAL AUDIENCES}

S Horvath. Radiation Safety Institute of Canada Toronto, Canada

\subsection{6/oemed-2018-ICOHabstracts.208}

Introduction An organisation must have a communications strategy that is actionable and understands their stakeholders, helps you converse with them, about the things they care about, in the places or platforms where they are already 'hanging out'. Budget, content and vision will not determine your success, instead, organisations must transform their fundamental communication concepts and modify them for this new two-way, end-user empowered medium of Social Media. The organisation's OSH content now must not only drive engagement, but also define whom you want to listen to and communicate with.

This presentation will provide a framework for establishing a social media strategy for $\mathrm{OSH}$ and nongovernmental organisations (NGO) to effectively integrate traditional and digital communication strategies and platforms to increase global outreach and access non-traditional and multi-cultural audiences.

It will present innovative approaches to accessing and leveraging new social media channels to create 'communities of interest' in OSH, expand professional networks and increase both research translation and knowledge translation. This discussion will use case studies of organisations increasing impact 
of information using digital communication tools like 'immersive content' to increase comprehension and understanding for multicultural or non-traditional audiences.

Methods Some key areas to be addressed include:

- Adopting an appropriate social media strategy

- Build a content plan

- Building your OSH message

- Choose your channels

- Building communities of interest

- Research data discussion from focus groups of millennials on OSH education

- Research data discussion on leadership and culture transformation

Results This presentation prevents evidence promoting the need for a strong OSH content plan that will deliver engaging materials and aligns with overall messaging and appropriate channels selected for use.

Conclusion This strategy cannot be achieved in isolation, but has to be integrated in parallel with all other traditional communication channels and in concert with an overall communication plan, and create a culture to nurture and sustain a social media transformation.

\section{2j CAMPAIGNS ARE NOT A SCIENCE. WHY? BECAUSE IT'S THE STORIES THAT MOVE THE WORLD}

M Holder. British Safety Council, London, UK

\subsection{6/oemed-2018-ICOHabstracts.209}

Introduction Speak Up Stay Safe is a British Safety Council campaign that seeks to show young people - and those that work or volunteer with young people - that OSH should be taken seriously but is an enabler to good work and successful activities. In doing so we have learnt that campaigning requires stories to produce memorable messages that promote real change. All too often OSH campaigns simply describe a problem and give little time to produce imaginative idea on what to do about it. Questions of narrative, language and image should be considered in OSH messages.

Methods Speak Up Stay Safe starts from the premise that as the audience is intended to be young people, they should be at the heart of the campaign and feature in its content and design. The campaign has taken a flexible approach by trying things out to see what works, for example by extending the campaign to include a media competition or the voluntary sector where there is concern that $\mathrm{OSH}$ can dissuade adults from volunteering with young people.

Results Results are measured through site visits (including on YouTube), retweets and Facebook likes. We have seen the campaign has become an important source of induction for young workers and has also provided opportunities for existing youth networks to introduce OSH in a way that makes sense to them.

Conclusion An inventive use of language and image is becoming even more important in making content more visible - if 'Google robots' pick up repetition and dull language, then it will be downgraded and information will become less visible. Variety, relevance, linkage and a clear message all contribute to the campaign that has seen it grow beyond the UK. One of the campaign videos has also contributed to a different campaign that is highlighting the mental health challenges for construction workers - which again is a testament to the flexible nature of the campaign.

\section{$1682 \mathrm{k}$ DIGITAL TRANSFORMATION. HOW IS IT CHANGING THE COMMUNICATION PARADIGM?}

S Vaxelaire. French Ministry of Agriculture, Paris, France

10.1136/oemed-2018-ICOHabstracts.210

Introduction The goal of this presentation is to better understand how digital transformation upsets the communication activity and drives public communication to reinvent itself in order to win viewer's attention and consequently rethink its strategy according to viewer's interest.

Methods Within a specific French Government digital strategy, the new rules for both Media and communicators have been collected and a new public communication strategy has been defined taking place in the updated Social Media landscape. We used some new tools to improve the surveillance of the 'bad buzz' strengthened by new feedback reports. We also created a Vision Camp event with our stakeholders.

Results We were able to provide a new framework in order to test any Social Media opportunity with 'newsroom' management. The Social Media activity came from the dark our funny side to a known and professional story with some strong results - from the live surveillance to the top of the trending topic. Our Social Media strategy was quoted by the French Government and we implemented some training courses. We also scheduled some hackathons, based on the Vision Camp, with both High Graduate Schools, startups and Research Institutes.

Conclusion Digital transforms the communication strategies with some specific audience typologies. For communicators, this is a new paradigm which redefines both strategy and communication actions. Communicators have to adapt themselves in order to create, disseminate and promote in a new way, thinking influencers and public first highlighted by native video contents. This information circulation and accelerating questions the media and so the press relations role and position.

\section{INFLUENCING SEARCH ENGINE OPTIMISATION TO DRIVE AUDIENCES TO OUR INFORMATION. LESSONS LEARNED FROM GOOGLE, BING, WEIBO, AND YAHOO}

M Lum. National Institute for Occupational Safety and Health, Washington, D.C., USA

\subsection{6/oemed-2018-ICOHabstracts.211}

Introduction Search engines have become the global gateway for information. Despite the expanded use of search engines there is little evidence of how governments and quasi-government organisations are optimising their search engine strategies to guide searchers to their safety and health information. The objective of this presentation is to discuss our findings summarised as the seven cardinal factors of search engine optimisation.

Methods Within a specific NIOSH program we collected data and performed a series of basic content analysis reviews and extracted a range of metrics including but not limited to what specific actions resulted in changes of page rank for selected topic pages, the effect of selected referring domains, the importance of authority and the impact of social media 